\title{
A hyperbolic model of two-phase flow: global solutions for large initial data
}

\author{
Debora Amadori, Paolo Baiti*, Andrea Corli \\ and Edda Dal Santo
}

\begin{abstract}
The paper deals with a simple nonlinear hyperbolic system of conservation laws modeling the flow of an inviscid fluid. The model is given by a standard isothermal $p$-system of the gasdynamics, for which phase transitions of the fluid are taken into consideration via a third homogeneous equation. We focus on the case of initial data consisting of two different phases separated by an interface. By means of an adapted version of the front tracking algorithm, we prove the global-in time existence of weak entropic solutions under suitable assumptions on the (possibly large) initial data.
\end{abstract}

Keywords: hyperbolic systems of conservation laws, phase transitions, wave-front tracking algorithm.

Mathematical subject classification: 35L65, 35L60, 35L67, 76T99.

\section{Introduction}

We consider the Cauchy problem related to the following nonlinear hyperbolic system of conservation laws

$$
\left\{\begin{array}{ll}
v_{t}-u_{x} & =0, \\
u_{t}+p(v, \lambda)_{x} & =0, \\
\lambda_{t} & =0,
\end{array} \quad t>0, x \in \mathbb{R},\right.
$$

and initial data of the special form

$$
\begin{gathered}
U_{o}(x)=\left(v_{o}(x), u_{o}(x), \lambda_{o}(x)\right), \\
\lambda_{o}(x)=\left\{\begin{array}{ll}
\lambda_{\ell} & \text { if } x<0, \\
\lambda_{r} & \text { if } x>0,
\end{array} \quad \lambda_{\ell} \neq \lambda_{r} \in[0,1] .\right.
\end{gathered}
$$

Received 27 March 2015.

*Corresponding author. 
System (1.1) models the one-dimensional flow of an inviscid fluid and is derived from [8]. The variable $v>0$ represents the specific volume, while $u$ is the velocity of the fluid. A third variable $\lambda \in[0,1]$ denoting the mass-density fraction of vapor in the fluid is meant to allow for the coexistence of different phases in the fluid: in particular, $\lambda=0$ characterizes the liquid phase and $\lambda=1$ the vapor one. A pressure law is fixed by $p(v, \lambda)=a^{2}(\lambda) / v$, where $a$ is a $\mathbf{C}^{\mathbf{1}}$ function defined on $[0,1]$ and satisfying $a(\lambda)>0$ for every $\lambda \in[0,1]$. We denote a solution $U=(v, u, \lambda) \in \Omega \doteq] 0, \infty[\times \mathbb{R} \times[0,1]$.

The global existence of weak solutions to the initial value problem for (1.1) and $\lambda_{o} \in \mathbf{B V}$ has already been proved in [5], under suitable assumptions on the total variation of the initial data. The general problem of [5] was specialized to the case of initial data of the form (1.2) in [1], whose results are partially presented in this paper. Such data give rise to a single contact discontinuity that is to be meant as an interface in the $(x, t)$-plane keeping the fluid into two separated phases. This leads to the study of two initial value problems for two isothermal $p$-systems [9], which are coupled through the interface at $x=0$, along which pressure and velocity coincide.

Being that the results in [5] clearly apply to this case, the focus in [1] is to weaken as much as possible the hypotheses allowing for larger initial data. Since, for system (1.1), the Riemann problem is solvable for any pair of initial states in $\Omega$, it would be interesting to prove whether global solutions exist just assuming that the initial data for $v, u$ belong to $\mathbf{B V}(\mathbb{R})$, without any other restriction, as it is the case for constant $\lambda$, see [9].

The plan of the paper is the following. The main result is stated in Theorem 2.1 and in Section 2 we briefly review the main steps of the proof; for the details we refer to [1]. Among the several novelties introduced in [1], we mention the original treatment of non-physical waves in the front tracking algorithm and the introduction of a Glimm functional that better accounts for interactions with the single contact discontinuity. In Section 3 we discuss some possible generalizations. First, we show that the result of [1] can be improved by adopting a more accurate Glimm functional provided that two special Riemann solvers are employed in the construction. At last, we see that a similar strategy applies to the case of initial data when $\lambda_{o}$ is piecewise constant with two jumps, see [2].

\section{An Existence Theorem}

In this section, we state our existence theorem and recall some basic facts on system (1.1). Given initial data (1.2), define

$$
p_{o}(x) \doteq p\left(v_{o}(x), \lambda_{o}(x)\right), a_{r}=a\left(\lambda_{r}\right) \text { and } a_{\ell}=a\left(\lambda_{\ell}\right) .
$$


The strength $\delta_{2}$ of the contact discontinuity located at $x=0$ is defined by

$$
\left.\delta_{2}=2 \frac{a_{r}-a_{\ell}}{a_{r}+a_{\ell}} \in\right]-2,2[.
$$

Theorem 2.1. Consider (1.1) with $p(v, \lambda)=a^{2}(\lambda) / v$ and initial data (1.2) satisfying $v_{o}(x) \geq \underline{v}>0$, for some constant $\underline{v}$. There exists a strictly decreasing function $\mathcal{K}:[0,2[\rightarrow \overline{\mathbb{R}}, \mathcal{K}(0)=+\infty$, with limit

$$
\lim _{r \rightarrow 2-} \mathcal{K}(r)=\frac{2}{3} \log (2+\sqrt{3})
$$

such that, if $\delta_{2} \neq 0$ and

$$
\mathrm{TV}\left(\log \left(p_{o}\right)\right)+\frac{1}{a_{\ell}} \underset{x<0}{\mathrm{TV}}\left(u_{o}\right)+\frac{1}{a_{r}} \underset{x>0}{\operatorname{TV}}\left(u_{o}\right)<\mathcal{K}\left(\left|\delta_{2}\right|\right),
$$

then the Cauchy problem (1.1), (1.2) has a weak entropic solution $U=(v, u, \lambda)$ defined for $t \in[0,+\infty)$.

Theorem 2.1 improves the main result obtained in [5] when applied to the case of a single contact discontinuity, as we show at the end of the section.

We now give the idea of the proof. For simplicity, we consider only the case $a_{\ell}<a_{r}$, hence $\delta_{2}>0$. Under the assumptions stated above, system (1.1) is strictly hyperbolic in $\Omega$, with two genuinely nonlinear characteristic fields of family 1 and 3 and a linearly degenerate one of family 2 . The special form of the data considered gives rise to a single contact discontinuity, which is a stationary (i.e with zero speed) 2-wave traveling along $x=0$; waves of family 1 or 3 are called sonic waves.

The strength $\epsilon_{i}$ of an $i$-wave, $i=1,2,3$, connecting a left state $U_{-}=$ $\left(v_{-}, u_{-}, \lambda_{-}\right)$to a right state $U_{+}=\left(v_{+}, u_{+}, \lambda_{+}\right)$is defined by

$$
\epsilon_{1}=\frac{1}{2} \log \frac{v_{+}}{v_{-}}, \quad \epsilon_{3}=\frac{1}{2} \log \frac{v_{-}}{v_{+}}, \quad \epsilon_{2}=2 \frac{a\left(\lambda_{+}\right)-a\left(\lambda_{-}\right)}{a\left(\lambda_{+}\right)+a\left(\lambda_{-}\right)}=2 \frac{a_{+}-a_{-}}{a_{+}+a_{-}} ;
$$

the size of the wave is defined by $\left|\epsilon_{i}\right|$.

In order to prove Theorem 2.1 we exploit a suitable wave-front tracking scheme. We use two different ways of solving the Riemann problems arising at interactions: an Accurate solver and a Simplified solver [7]. More precisely, at time 0 as well as at times $>0$ where an interaction between sonic waves occurs, we use the Accurate solver. As for interactions involving the single 2-wave, if the size of the incoming wave is larger than a positive threshold $\rho$ we use again 
the Accurate procedure, otherwise we use the Simplified solver. Such a trick is needed to control the total number of fronts and interactions in the approximate solutions. In the Simplified solver, the standard approach [7] consists in letting the waves pass through each other and in collecting all the errors coming from their commutation into a single so-called non-physical front traveling with a fixed positive speed greater than all characteristic speeds. Here, instead, we choose to assign zero speed to the emerging non-physical waves and attach them to the front $x=0$. Indeed, in the case of system (1.1) a non-physical wave changes neither the side values of $v$ nor those of $\lambda$; on the contrary, a 2-wave does not change the side values of $u$ [5]. Thus, we can define a new wave by composing the 2-wave with a non-physical wave. Notice that the order of composition does not matter, because a 2 -wave and a non-physical wave act on different state variables. More precisely, the resulting composite $(2,0)$-wave curve through the point $U_{o}=\left(v_{o}, u_{o}, \lambda_{\ell}\right)$ is given by

$$
u \mapsto\left(\left(a_{r}^{2} / a_{\ell}^{2}\right) v_{o}, u, \lambda_{r}\right) .
$$

The strength of a composite wave is defined by $\delta_{2,0}=u-u_{o}$ and measures only the variation of its $u$-component (recall that the quantity $\delta_{2}$ remains constant at any interaction with 1 - or 3-waves [5]). Finally, notice that a $(2,0)$-wave reduces to a 2-wave as long as non-physical waves are missing.
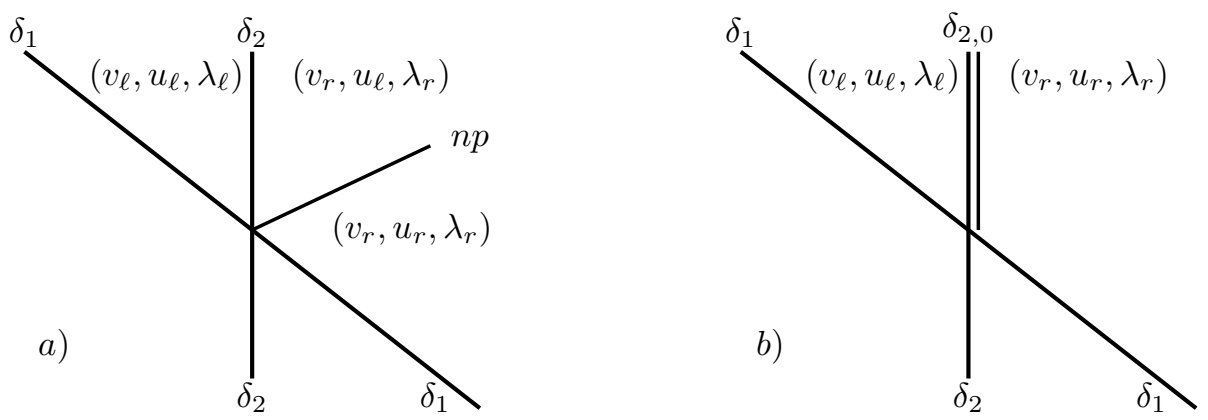

Figure 1: The interaction between a 1- and a 2-wave that generates a 1-wave and a 2-wave of the same strength of the incoming ones and: $a$ ) a standard non-physical wave; $b)$ a $(2,0)$-composite wave.

Consequently, we introduce two new Riemann solvers, that we call Pseudo Accurate solver and Pseudo Simplified solver and that allow us to uniquely solve every Riemann problem in terms of 1-, 3- and composite waves; see Figure 1 $b$ ) and [1, Propositions 3.1 and 3.2] for rigorous definitions. A first algorithmic 
advantage is given by the fact that any interaction involves definitively only two incoming and two outgoing fronts, hence a bound on the total number of fronts and interactions immediately follows. Moreover, both the new solvers satisfy the same interaction estimates presented in [5].

Lemma 2.2. Assume that a wave $\delta_{i}, i=1,3$, interacts with $\delta_{2,0}$.

If the Riemann problem is solved by the Pseudo Accurate solver, then the strengths $\epsilon_{i}$ of the outgoing waves satisfy $\epsilon_{2,0}=\delta_{2,0}$ and

$$
\begin{aligned}
& \left|\epsilon_{i}-\delta_{i}\right|=\left|\epsilon_{j}\right| \leq \frac{1}{2} \delta_{2}\left|\delta_{i}\right|, \quad i, j=1,3, i \neq j, \\
& \left|\epsilon_{1}\right|+\left|\epsilon_{3}\right| \leq \begin{cases}\left|\delta_{1}\right|+\delta_{2}\left|\delta_{1}\right| & \text { if } i=1, \\
\left|\delta_{3}\right| & \text { if } i=3 .\end{cases}
\end{aligned}
$$

If the Riemann problem is solved by the Pseudo Simplified solver and we assume $\left|\delta_{i}\right|<m$, then there exists $C_{o}=C_{o}(m)$ such that

$$
\left|\epsilon_{2,0}-\delta_{2,0}\right| \leq C_{o} \delta_{2}\left|\delta_{i}\right|
$$

Next, let $\gamma_{i}(i=1,3)$ denote the strengths of the $i$-waves and $\gamma_{2,0}$ that of the composite wave in a front tracking approximate solution, at some time $t$ at which no interaction occurs. For suitable constants $\xi \geq 1, K_{n p}>0, K \geq 1$, we define the functionals

$$
\begin{aligned}
L & =\sum_{\substack{i=1,3 \\
\gamma_{i}>0}}\left|\gamma_{i}\right|+\xi \sum_{\substack{i=1,3 \\
\gamma_{i}<0}}\left|\gamma_{i}\right|+K_{n p}\left|\gamma_{2,0}\right|, \quad \bar{L}=\sum_{i=1,3}\left|\gamma_{i}\right|, \\
V & =\sum_{\substack{i=1,3 \\
\gamma_{i}>0, \mathcal{A}}}\left|\gamma_{i}\right|+\xi \sum_{\substack{i=1,3 \\
\gamma_{i}<0, \mathcal{A}}}\left|\gamma_{i}\right|, \quad Q=\delta_{2} V, \\
F & =L+K Q .
\end{aligned}
$$

The summation in $V$ is performed only over the set $\mathcal{A}$ of waves approaching the front at $x=0$, i.e. the waves of the family 1 (and 3) located at the right (left, respectively) of $x=0$. Remark that the functional (2.9) is equivalent to the total variation of the approximate solutions and is not the same of [5, (5.1)], since here the parameter $\xi$ appears also in $V$ and $Q$. By carefully analyzing how the functionals $L, V$ vary across interaction times, we find that under certain conditions on $\xi, K_{n p}, K$, the functional $F$ decreases and the total variation of the 
approximate solutions is bounded. This proves that the algorithm is well-defined and generates a sequence of $\epsilon$-approximate wave-front tracking solutions in the sense of [7]. Then, the following fundamental result holds.

Proposition 2.3 (Global decreasing of $F$ ). Let $m>0$ and assume that

$$
\bar{L}(0+) \leq m c(m), \quad c(m) \doteq \frac{\cosh m-1}{\cosh m+1} .
$$

Then, every shock wave generated by the algorithm has strength bounded by $m$ and there exist constants $\xi, K, K_{n p}$ such that $F$ is decreasing for every $t>0$.

The function $c(z)$ works as a damping coefficient for the reflected wave in the interactions between sonic waves of the same family. Moreover, it is proved [1] to be deeply connected to the coefficients in the reflection matrices used in the stability analysis of the Riemann problems for system $(1.1)$, see $[4,10]$.

Since it holds

$$
\bar{L}(0+) \leq \frac{1}{2} \mathrm{TV}\left(\log \left(p_{o}\right)\right)+\frac{1}{2 a_{\ell}} \operatorname{TV}_{x<0}\left(u_{o}\right)+\frac{1}{2 a_{r}} \operatorname{TV}_{x>0}\left(u_{o}\right),
$$

then Proposition 2.3 and a standard compactness argument allow us to pass to the limit in the sequence, thus proving Theorem 2.1.

To conclude the section, we make a comparison between Theorem 2.1 and the existence result obtained in [5]. There, the analogous of (2.3) (reformulated as in $[6,(3.7)])$ is given by

$$
\mathrm{TV}\left(\log \left(p_{o}\right)\right)+\frac{1}{a_{\ell}} \underset{x<0}{\mathrm{TV}}\left(u_{o}\right)+\frac{1}{a_{r}} \underset{x>0}{\mathrm{TV}}\left(u_{o}\right)<H\left(\left|\delta_{2}\right|\right),
$$

where the function $H(r)$ is only defined for $r<1 / 2$ by

$$
H(r) \doteq 2(1-2 r) k^{-1}(r), \quad r=k(z) \doteq \frac{1-\sqrt{d(z)}}{2-\sqrt{d(z)}} .
$$

Here above, $d(z)$ is the damping coefficient introduced in [5, Lemma 5.6]. Hence, the result of Theorem 2.1 is completely new for $1 / 2 \leq\left|\delta_{2}\right|<2$, including the case where the 2-wave may be arbitrarily large, i.e. $\left|\delta_{2}\right|$ close to 2. Since $\mathcal{K}(r)>H(r)$ for $0 \leq r<1 / 2$ (see Figure 2), Theorem 2.1 improves the result of [5] even in the common range $\left|\delta_{2}\right|<1 / 2$. 


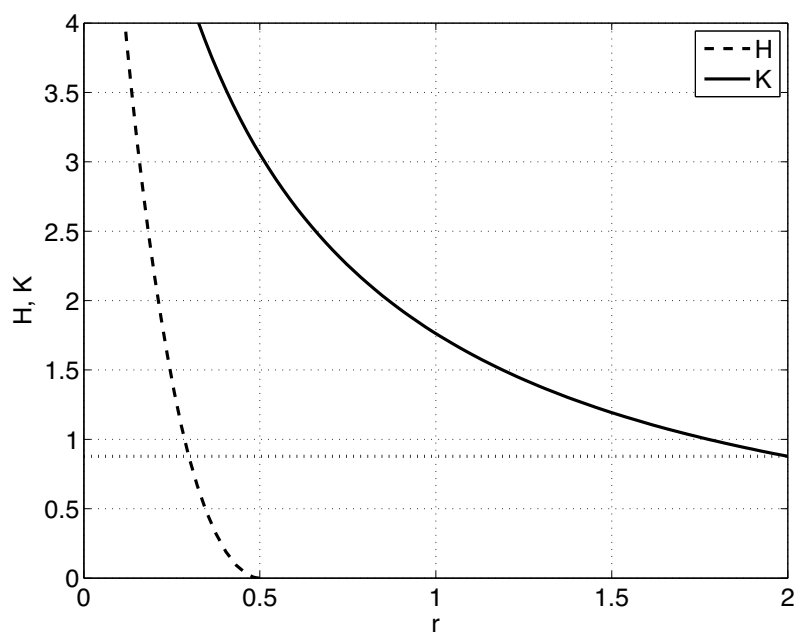

Figure 2: The functions $H$ (dashed line) and $\mathcal{K}$ (solid line). The horizontal dotted line gives the asymptotic value $2 / 3 \log (2+\sqrt{3})$ of $\mathcal{K}$ for $r \rightarrow 2-$.

\section{Further results}

The results presented in Section 2 can be generalized in several directions. First, we discuss a possible improvement of Theorem 2.1. Notice that the only case in which the functionals $L$ and $\bar{L}$ in (2.8) can increase is when a 1-wave interacts with the 2-wave: indeed, they do not increase for interactions between sonic waves ([1, Lemma 5.4] or [9]) and the same happens for interactions between a 3 -wave and the 2 -wave by (2.6). This suggests to let the 3 -waves (especially 3 shocks) out of the definition of $V$, since they are of no use to control the possible increase of $L$. Unfortunately, if we were to modify the definition of $V$ by just removing 3 -shocks as follows

$$
\tilde{V}=\sum_{\substack{i=1,3 \\ \gamma_{i}>0, \mathcal{A}}}\left|\gamma_{i}\right|+\xi \sum_{\substack{i=1 \\ \gamma_{i}<0, \mathcal{A}}}\left|\gamma_{i}\right|, \quad \tilde{Q}=\delta_{2} \tilde{V}, \quad \tilde{F}=L+K \tilde{Q},
$$

we would obtain a new functional $\tilde{F}$ which is not always decreasing along approximate solutions. Indeed, $\tilde{F}$ does decrease at interactions solved by the Pseudo Accurate solver, but this is no longer true for interactions between 3shocks and the composite wave solved by the Pseudo Simplified solver. The breakdown is due to the fact that the Simplified procedure prescribes for the outgoing physical waves to have exactly the same sizes of the incoming ones: this 
choice is clearly inadequate in the current situation, since in the interaction of a 3-wave with a 2-wave the size of the real transmitted wave strictly decreases. Then, the idea is to intervene in the definition of the Pseudo Simplified solver in order to find a better balancing of the size of the transmitted wave and that of the non-physical error attached to the composite $(2,0)$-wave. In $[2,3]$ this is achieved by taking as non-physical errors non-entropic waves associated to integral curves of (1.1) and formally computed as reflected waves. In brief, we introduce two modified Riemann solvers to construct the approximate solutions and we find that $\tilde{F}$ decreases along them. In this way, we prove the following result.

Theorem 3.1. Under the same hypotheses of Theorem 2.1, if either $\delta_{2}>0$ and the initial data satisfy

$$
\begin{gathered}
\frac{1}{1+\left|\delta_{2}\right|}\left[\underset{x<0}{\operatorname{TV}}\left(\log \left(p_{o}\right)\right)+\frac{1}{a_{\ell}} \underset{x<0}{\left.\operatorname{TV}\left(u_{o}\right)\right]}\right. \\
+\left[\underset{x>0}{\operatorname{TV}}\left(\log \left(p_{o}\right)\right)+\frac{1}{a_{r}} \underset{x>0}{\operatorname{TV}}\left(u_{o}\right)\right]<\mathcal{K}\left(\left|\delta_{2}\right|\right),
\end{gathered}
$$

or $\delta_{2}<0$ and the initial data satisfy

$$
\begin{gathered}
{\left[\operatorname{TV}_{x<0}\left(\log \left(p_{o}\right)\right)+\frac{1}{a_{\ell}} \underset{x<0}{\operatorname{TV}}\left(u_{o}\right)\right]} \\
+\frac{1}{1+\left|\delta_{2}\right|}\left[\operatorname{TV}_{x>0}\left(\log \left(p_{o}\right)\right)+\frac{1}{a_{r}} \operatorname{TV}_{x>0}\left(u_{o}\right)\right]<\mathcal{K}\left(\left|\delta_{2}\right|\right),
\end{gathered}
$$

then the Cauchy problem related to (1.1) has a global weak entropic solution $U=(v, u, \lambda)$.

Remark that Theorem 3.1 improves Theorem 2.1, since the total variation of the initial data (thanks to the coefficient of the first term in (3.1) or of the second term in (3.2)) can be taken larger in the more liquid region (i.e. $x<0$ if $\delta_{2}>0$ or $x>0$ if $\delta_{2}<0$ ).

More generally, a similar analysis can be performed for a Cauchy problem associated to (1.1) and an initial datum for $\lambda$ piecewise constant with two (or even more) jumps, i.e. of the form

$$
\begin{gathered}
U_{o}(x)=\left(v_{o}(x), u_{o}(x), \lambda_{o}(x)\right), \\
\lambda_{o}(x)=\left\{\begin{array}{ll}
\lambda_{\ell} & \text { if } x<a, \\
\lambda_{m} & \text { if } a<x<6, \\
\lambda_{r} & \text { if } x>6,
\end{array} \quad \lambda_{\ell} \neq \lambda_{m} \neq \lambda_{r} \in[0,1] .\right.
\end{gathered}
$$


For simplicity, let $a_{\ell}=a\left(\lambda_{\ell}\right), a_{m}=a\left(\lambda_{m}\right), a_{r}=a\left(\lambda_{r}\right)$ and, for example, consider the case studied in [2]

$$
a_{m}<\min \left\{a_{\ell}, a_{r}\right\} .
$$

If $a$ is assumed to be increasing, then (3.4) implies $\lambda_{m}<\min \left\{\lambda_{\ell}, \lambda_{r}\right\}$. Roughly speaking, this case can be thought of as representing a liquid drop surrounded by a gaseous environment, since it corresponds to three homogeneous mixtures in the fluid, the more liquid of which is in the interval $] a, b[$. Initial data satisfying (3.3) give rise to two contact discontinuities propagating along $x=a$ and $x=6$ in the $(x, t)$-plane, respectively. Their strengths are defined as [2]

$$
\eta=2 \frac{a_{m}-a_{\ell}}{a_{m}+a_{\ell}}, \quad \zeta=2 \frac{a_{r}-a_{m}}{a_{r}+a_{m}} .
$$

Notice that by (3.4) we have $\eta<0, \zeta>0$ and $|\eta|,|\zeta| \in[0,2[$. In order to prove existence of weak solutions, we need that the 2-waves $\eta$ and $\zeta$ satisfy the stability condition

$$
\max \left\{\left(1+\frac{|\zeta|}{2}\right) \frac{|\eta|}{2},\left(1+\frac{|\eta|}{2}\right) \frac{|\zeta|}{2}\right\}<1,
$$

which identifies a domain $\mathcal{D} \subset[0,2[\times[0,2[$, see Figure 3 . Such a set is where we define a non-negative and continuous function $\mathcal{H}$ given by

$$
\mathcal{H}(|\eta|,|\zeta|) \doteq \max \left\{\frac{|\zeta|}{1-(1+|\zeta| / 2)|\eta| / 2}, \frac{|\eta|}{1-(1+|\eta| / 2)|\zeta| / 2}\right\} .
$$

In this framework, an existence theorem analogous to Theorem 2.1 holds.

Theorem 3.2. Consider initial data (3.3) with $v_{o}(x) \geq \underline{v}>0$, for some constant $\underline{v}$. Assume also (3.4) and (3.6). There exists a strictly decreasing function $\mathcal{K}$ : $[0,+\infty[\rightarrow \overline{\mathbb{R}}, \mathcal{K}(0)=+\infty$, with

$$
\lim _{r \rightarrow+\infty} \mathcal{K}(r)=0
$$

such that if it holds

$$
\begin{gathered}
\operatorname{TV}_{x<a}\left(\log \left(p_{o}\right), \frac{u_{o}}{a_{\ell}}\right)+\frac{1}{1+\mathcal{H}(|\eta|,|\zeta|)} \underset{a<x<6}{\operatorname{TV}}\left(\log \left(p_{o}\right), \frac{u_{o}}{a_{m}}\right) \\
+\operatorname{TV}_{x>6}\left(\log \left(p_{o}\right), \frac{u_{o}}{a_{r}}\right)<\mathcal{K}(\mathcal{H}(|\eta|,|\zeta|)),
\end{gathered}
$$




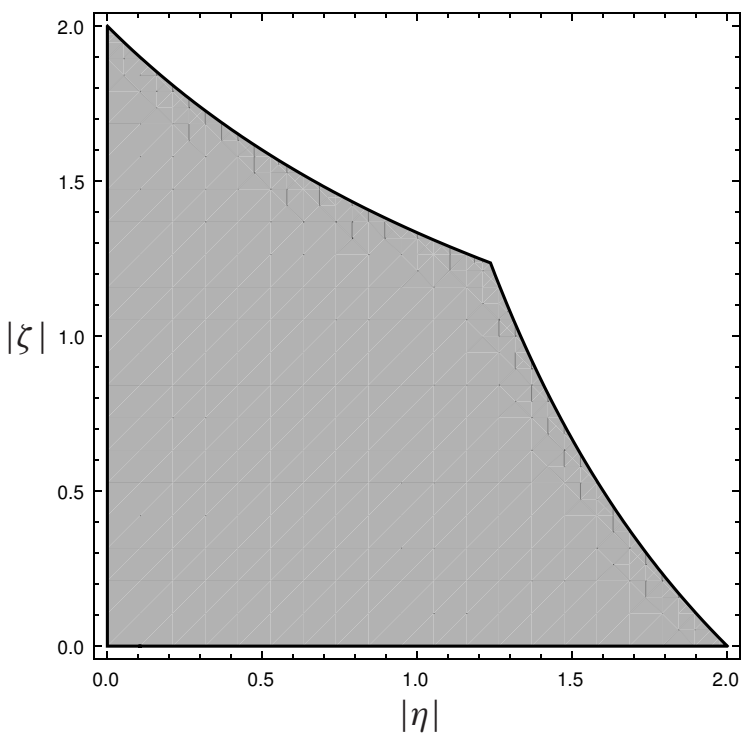

Figure 3: The domain $\mathcal{D}$ in the $(|\eta|,|\zeta|)$-plane.

then the Cauchy problem (1.1), (3.3) has a weak entropic solution $(v, u, \lambda)$ defined for $t \in[0,+\infty[$.

Notice that $\mathcal{H}=0$ only when $\eta=\zeta=0$; moreover, we have $\mathcal{H}(|\eta|, 0)=|\eta|$ and $\mathcal{H}(0,|\zeta|)=|\zeta|$ and we can recover the result obtained for the single 2-wave case.

\section{References}

[1] D. Amadori, P. Baiti, A. Corli and E. Dal Santo. Global weak solutions for a model of two-phase flow with a single interface. J. Evol. Equ., 15(3) (2015), 699-726.

[2] D. Amadori, P. Baiti, A. Corli and E. Dal Santo. Global existence of solutions for a multi-phase flow: a drop in a gas-tube. Submitted (2015).

[3] D. Amadori, P. Baiti, A. Corli and E. Dal Santo. Global existence of solutions for a multi-phase flow: a bubble in a liquid tube and related cases. Acta Math. Sci. Ser. B 35(4) (2015), 832-854.

[4] D. Amadori and A. Corli. A hyperbolic model of multi-phase flow. In S. BenzoniGavage and D. Serre, editors, Hyperbolic Problems: Theory, Numerics, Applications, pages 407-414. Springer (2008).

[5] D. Amadori and A.Corli. On a model of multiphase flow. SIAM J. Math. Anal., 40(1) (2008), 134-166.

[6] D. Amadori and A. Corli. Global existence of BV solutions and relaxation limit for a model of multiphase reactive flow. Nonlinear Anal., 72(5) (2010), 2527-2541. 
[7] A. Bressan. Hyperbolic systems of conservation laws. Oxford University Press (2000).

[8] H. Fan. On a model of the dynamics of liquid/vapor phase transitions. SIAM J. Appl. Math., 60(4) (2000), 1270-1301.

[9] T. Nishida. Global solution for an initial boundary value problem of a quasilinear hyperbolic system. Proc. Japan Acad., 44 (1968), 642-646.

[10] S. Schochet. Sufficient conditions for local existence via Glimm's scheme for large BV data. J. Differential Equations, 89(2) (1991), 317-354.

\section{Debora Amadori}

Department of Engineering and Computer Science and Mathematics

University of L'Aquila

ITALY

E-mail: amadori@univaq.it

\section{Paolo Baiti and Edda Dal Santo}

Department of Mathematics and Computer Science

University of Udine

ITALY

E-mails: paolo.baiti@uniud.it; edda.dalsanto@uniud.it

\section{Andrea Corli}

Department of Mathematics and Computer Science

University of Ferrara

ITALY

E-mail: andrea.corli@unife.it 